
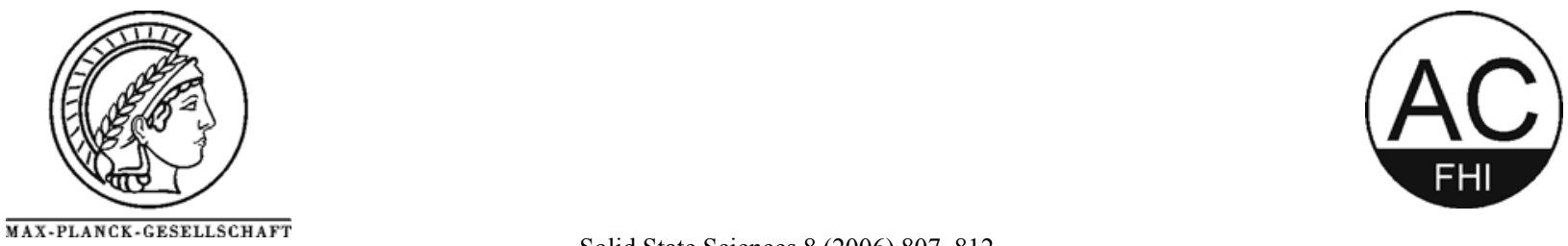

Solid State Sciences 8 (2006) 807-812

\title{
The crystal structure of $\varepsilon-\mathrm{VOPO}_{4}$
}

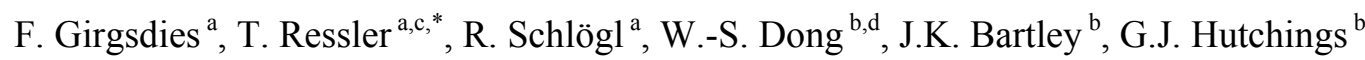

${ }^{a}$ Department of Inorganic Chemistry, Fritz-Haber-Institut der Max-Planck-Gesellschaft, Faradayweg 4-6, D-14195 Berlin, Germany

${ }^{\mathrm{b}}$ School of Chemistry, Cardiff University, Main Building, Park Place, Cardiff, UK CF10 3AT, Great Britain

${ }^{\mathrm{c}}$ Current address: Technische Universität Berlin, Institut für Chemie, Sekr. C2, Straße des 17. Juni 135,

D-10623 Berlin, Germany

${ }^{\mathrm{d}}$ Current address: School of Chemistry \& Materials, Shanxi Normal University, Xi'an 710062, China

* Corresponding author: Tel.: +49 30314 79736; fax: +49 3031421106.

Received: 22 December 2005; received in revised form: 31 March 2006; accepted: 18 April 2006

\begin{abstract}
The crystal structure of $\varepsilon-\mathrm{VOPO}_{4}$ was determined in the space group Cc from X-ray powder diffraction data using a rigid body approach. The resulting structure is compared to a recently published, slightly different structure model (space group P $2_{1} / \mathrm{n}$ ) using Rietveld refinement. It was found that the new $\mathrm{Cc}$ model consistently yields a better fit to the observed data and exhibits a less distorted, more stable geometry. The crystal structure of $\varepsilon-\mathrm{VOPO}_{4}$ is discussed in comparison to $\beta-\mathrm{VOPO}_{4}$, monoclinic $\mathrm{VPO}_{4} \cdot \mathrm{H}_{2} \mathrm{O}$, and other related structures.
\end{abstract}

Keywords: vanadium; phosphate; crystal structure; powder diffraction; ab initio structure determination; rigid body constraints;

\section{Introduction}

The numerous polymorphs of $\mathrm{VOPO}_{4}$ have attracted much attention among both catalysis researchers and electrochemists. The phases $\alpha_{\mathrm{II}^{-}}, \delta$ - and $\gamma-\mathrm{VOPO}_{4}$ have been reported to be present in activated $(\mathrm{VO})_{2} \mathrm{P}_{2} \mathrm{O}_{7}$ catalysts for the partial oxidation of $n$-butane to maleic anhydride $[1,2]$. Thus, they may be related to the activity of the catalyst [3]. In lithium battery research, $\delta$ - and especially $\varepsilon-\mathrm{VOPO}_{4}$, the most recently discovered polymorph [4], show promising electrochemical properties [5-8]. In both fields of research, knowledge of the crystal structure of the respective phases is desirable, since it is a prerequisite to uncover structure(re)activity relationships. Despite all efforts reflecting this interest, the knowledge about the structures of several $\mathrm{VOPO}_{4}$ polymorphs is still limited. The main reason is that many of the polymorphs are difficult to synthesize as single phase materials of reasonable crystallinity, combined with the inherent problems of crystal structure solution from powder diffraction data.
Since the discovery of $\varepsilon-\mathrm{VOPO}_{4}$ by Lim et al., it was argued that its structure should be both similar to $\beta-\mathrm{VOPO}_{4}$ [9], the most stable polymorph, and related to monoclinic $\mathrm{VPO}_{4} \cdot \mathrm{H}_{2} \mathrm{O}$ [10], which can be reversibly converted to $\varepsilon-\mathrm{VOPO}_{4}$ [4]. This claim was further substantiated by recent work of Song et al., where a structure model derived from monoclinic $\mathrm{VPO}_{4} \cdot \mathrm{H}_{2} \mathrm{O}$ (space group $\mathrm{C} 2 / \mathrm{c}$ ) was refined successfully in the space group $P 2_{1} / n$ [8].

While investigating the potential of a rigid body approach in $a b$ initio structure determination from powder diffraction data for some of the $\mathrm{VOPO}_{4}$ polymorphs, we found a slightly different model (space group $C c$ ) for the structure of $\varepsilon-\mathrm{VOPO}_{4}$, which will be presented here.

\section{Experimental}

\subsection{Sample preparation}

Monoclinic $\mathrm{VPO}_{4} \cdot \mathrm{H}_{2} \mathrm{O}$ was prepared by the reduction of $\mathrm{VOHPO}_{4} \cdot 0.5 \mathrm{H}_{2} \mathrm{O}(2.0 \mathrm{~g})$ derived from the standard 
VPO route [11] with 1-octanol $(40 \mathrm{ml})$ in an autoclave at $250{ }^{\circ} \mathrm{C}$ for $24 \mathrm{~h}$ in $\mathrm{N}_{2}$. The precursor was recovered by filtration, washed with acetone, and dried at $110^{\circ} \mathrm{C}$ in air for $24 \mathrm{~h}$. The $\varepsilon-\mathrm{VOPO}_{4}$ was prepared by calcination of the as synthesized monoclinic $\mathrm{VPO}_{4} \cdot \mathrm{H}_{2} \mathrm{O}$ at $500{ }^{\circ} \mathrm{C}$ for $4 \mathrm{~h}$ in air.

\subsection{Diffraction data collection}

X-ray diffraction data were collected on a D8 ADVANCE powder diffractometer (Bruker AXS) in Theta/Theta reflection geometry. CuK $\alpha$ radiation from a $\mathrm{Cu}$ X-ray tube was selected secondarily by means of a SolX energy dispersive solid state detector (Baltic Instruments). The data acquisition was performed in six consecutive scans. After confirming that the diffraction pattern exhibited no change over the total acquisition time of 33 hours, the six data sets were averaged.

\subsection{Structure determination}

The program Topas [12] was used for $a b$ initio structure determination from powder diffraction data (SDPD). In a first step, a whole powder pattern decomposition (WPPD) was performed employing the Le Bail method. However, the purpose of this procedure was not to extract $h k l$ intensities, but to obtain reasonable background, profile, and lattice parameters. Since the diffraction data can be equally well explained using either a monoclinic or orthorhombic unit cell, both possibilities were tested separately. The starting lattice parameters were taken from reference [4]. As the correct space group was unknown, space groups without any systematic absences were chosen for the WPPD (P2/m for the monoclinic and Pmmm for the orthorhombic cell, respectively).

In a second step, the actual SPDP process was performed in direct space on the diffraction step intensity data [13], using fixed parameters obtained from previous refinement in the WPPD. To increase the chance of a successful structure solution, a rigid body approach was employed, i.e. the internal geometries of an idealized tetrahedral $\mathrm{PO}_{4}$ group and a linear $\mathrm{V}=\mathrm{O}$ fragment were kept fixed during the process, with bond lengths derived from the known crystal structure of $\beta-\mathrm{VOPO}_{4}$ (ICSD entry 9413) [9]. Only the translation and rotation of these two rigid bodies were subject to randomization and refinement in the simulated annealing process. A number of monoclinic and orthorhombic space groups were tested by trial and error. Space groups with systematic absences incompatible with the diffraction pattern were excluded a priori, as were some candidates that seemed geometrically unlikely (e.g. orthorhombic space groups with intersecting mirror planes). The results were evaluated according to both the $R_{w p}$ value and the plausibility of the resulting structure.

\subsection{Structure refinement}

All subsequent Rietveld structure refinements were also performed using the program Topas. In all cases, a common isotropic temperature factor was refined for all atoms. The presence of some $\beta-\mathrm{VOPO}_{4}$ was taken into account by adding this phase to the refinement (fixed atomic coordinates, lattice parameters refined, common isotropic temperature factor shared with $\left.\varepsilon-\mathrm{VOPO}_{4}\right)$. To avoid floating origin problems in the space group $C c$, the $x$ and $z$ coordinates of the $\mathrm{V}$ atom were fixed to arbitrary values $(x=z=1 / 2)$. All bond lengths and angles were calculated with Platon for Windows $[14,15]$. Structure drawings were created with Diamond [16].

\section{Results}

\subsection{Structure determination}

The XRD data revealed that the sample used was not a single-phase material. In addition to the diffraction pattern of $\varepsilon-\mathrm{VOPO}_{4}$, weak diffraction peaks typical for $\beta-\mathrm{VOPO}_{4}$ were observed, as well as a very weak unidentified peak at $21.23^{\circ} 2 \theta$. The intensities of the impurity reflections overlapping with $\varepsilon$ - $\mathrm{VOPO}_{4}$ peaks were assumed to be weak enough to be ignored in the initial structure solution step.

Among all the space groups tested in the structure solution attempts, only the space group Cc yielded a plausible structure model, which was also the solution with the lowest $R_{w p}$ value. This result confirms that the symmetry of the structure is indeed monoclinic, although the $a$ and $c$ lattice parameters are equal within a few standard deviations, rendering the unit cell pseudo-orthorhombic metrically. This situation results in a systematic overlap of $h k l$ and $l k h$ reflections, which severely reduces the data resolution and decreases the stability of the structure refinement, as was already indicated by Song et al. [8].

\subsection{Structure refinement}

To avoid stability problems, the refinement process was performed step-by-step, gradually increasing the number of free parameters. Thus, the rigid body constraints used in the structure solution process were first kept. After convergence, it was found that most atoms (i.e. the $\mathrm{V}=\mathrm{O}$ fragment and an O-P-O part of the $\mathrm{PO}_{4}$ unit) aligned very well in a common plane that corresponds to the mirror plane in the structure of $\beta-\mathrm{VOPO}_{4}$. This "pseudo-mirror" like arrangement readily explains why the $a$ and $c$ lattice parameters of the monoclinic cell are so similar to each other, despite the absence of a higher (orthorhombic) symmetry. Based on this observation, the rather restrictive rigid body model was exchanged for a more flexible "pseudomirror model". The following constraints were used: 
(i) lattice parameters $a$ and $c$ are equal,

(ii) above mentioned atoms lie exactly in one plane (the pseudo-mirror plane) by correlation of their $z$ to their $x$ coordinates, and the remaining two oxygen atoms are pseudo-mirror images of each other, i.e. their $x$ and $z$ coordinates are crosscorrelated while their $y$ coordinates are equal.
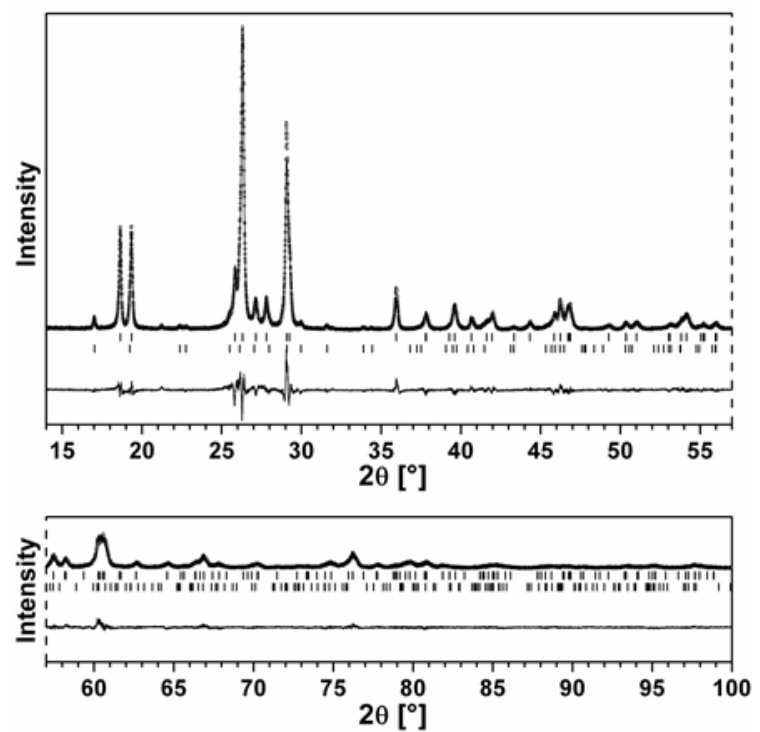

Figure 1: Comparison of observed (circles) and calculated diffraction pattern (continuous line) after final Rietveld refinement. For convenience, the display is split into lower and higher angle range panels (same intensity and angular scale). Calculated peak positions are indicated as tick marks (upper row: $\varepsilon$-VOPO${ }_{4}$, lower row: $\beta-\mathrm{VOPO}_{4}$ ). The continuous line below the tick marks represents the difference between observed and calculated pattern.

Table I: Crystal and experimental data for $\varepsilon-\mathrm{VOPO}_{4}$

\begin{tabular}{ll}
\hline Chemical formula & $\mathrm{VOPO}_{4}$ \\
Crystal system & monoclinic \\
Space group & $C c($ No. 9$)$ \\
$a(\AA)$ & $7.2659(3)^{\mathrm{a})}$ \\
$b(\AA)$ & $6.8934(2)^{\mathrm{a})}$ \\
$c(\AA)$ & $7.2651(3)^{\mathrm{a})}$ \\
$\beta\left({ }^{\circ}\right)$ & $115.3396(13)^{\mathrm{a})}$ \\
$V\left(\AA^{3}\right)$ & $328.88(2)^{\mathrm{a})}$ \\
$Z$ & 4 \\
$M_{r}\left(\mathrm{~g} \mathrm{~mol}^{-1}\right)$ & 161.91 \\
$\rho_{\text {calc }}\left(\mathrm{g} \mathrm{cm}^{-3}\right)$ & $3.2700(2)^{\mathrm{a})}$ \\
$\mu\left(\mathrm{cm}^{-1}\right)$ & $291.58(2)^{\mathrm{a})}$ \\
$2 \theta \mathrm{min}^{-\mathrm{max} / \mathrm{step}}\left(^{\circ}\right)$ & $14 / 100 / 0.005$ \\
$R_{p}$ & 0.1083 \\
$R_{w p}$ & 0.1390 \\
$R_{\text {exp }}$ & 0.0900 \\
$R_{\text {Bragg }}$ & 0.0302 \\
\hline
\end{tabular}

a) The uncertainties listed for the lattice constants (and parameters derived thereof) are given as provided by the program used. We think these values are surprisingly low and assume that the real errors are at least one order of magnitude larger.
Table II: Atomic coordinates for $\varepsilon-\mathrm{VOPO}_{4}$

\begin{tabular}{llllll}
\hline Atom & Site & $x / a$ & $y / b$ & $z / c$ & $\mathrm{~B}_{\text {iso }}$ \\
\hline $\mathrm{V}$ & $4 a$ & $0.5^{\text {a) }}$ & $0.2711(3)$ & $0.5^{\text {a) }}$ & $0.43(3)^{\mathrm{b})}$ \\
$\mathrm{O} 1$ & $4 a$ & $0.6867(16)$ & $0.3539(7)$ & $0.6913(15)$ & $0.43(3)^{\mathrm{b}}$ \\
$\mathrm{P}$ & $4 a$ & $0.2057(8)$ & $0.6197(4)$ & $0.2146(10)$ & $0.43(3)^{\mathrm{b})}$ \\
$\mathrm{O} 2$ & $4 a$ & $0.0463(11)$ & $0.4850(8)$ & $0.0539(13)$ & $0.43(3)^{\mathrm{b}}$ \\
$\mathrm{O} 3$ & $4 a$ & $0.3637(15)$ & $0.4984(9)$ & $0.3867(16)$ & $0.43(3)^{\mathrm{b}}$ \\
$\mathrm{O} 4$ & $4 a$ & $0.099(4)$ & $0.7595(11)$ & $0.309(3)$ & $0.43(3)^{\mathrm{b}}$ \\
$\mathrm{O} 5$ & $4 a$ & $0.310(4)$ & $0.7552(8)$ & $0.108(4)$ & $0.43(3)^{\mathrm{b}}$ \\
\hline
\end{tabular}

${ }^{\text {a) }}$ fixed (floating origin constraint)

${ }^{b)}$ common temperature factor

Table III: Selected bond lengths $[\AA]$ and angles $\left[{ }^{\circ}\right]$ for $\varepsilon-\mathrm{VOPO}_{4}$

\begin{tabular}{|c|c|c|c|}
\hline V-O1 & $1.572(11)$ & $\mathrm{V}-\mathrm{O} 1^{\mathrm{iv}}$ & $2.556(11)$ \\
\hline $\mathrm{V}-\mathrm{O} 2^{\mathrm{i}}$ & $1.861(6)$ & $\mathrm{V}-\mathrm{O} 3$ & $1.838(9)$ \\
\hline $\mathrm{V}-\mathrm{O} 4^{\mathrm{ii}}$ & $1.921(18)$ & $\mathrm{V}-\mathrm{O} 5^{\mathrm{iii}}$ & $1.88(2)$ \\
\hline $\mathrm{P}-\mathrm{O} 2$ & $1.528(9)$ & $\mathrm{P}-\mathrm{O} 3$ & $1.552(13)$ \\
\hline $\mathrm{P}-\mathrm{O} 4$ & $1.477(18)$ & P-O5 & $1.562(18)$ \\
\hline $\mathrm{O} 1-\mathrm{V}-\mathrm{O} 2^{\mathrm{i}}$ & $97.2(3)$ & O1-V-O3 & 101.1(4) \\
\hline $\mathrm{O} 1-\mathrm{V}-\mathrm{O} 4^{\mathrm{ii}}$ & $104.1(7)$ & $\mathrm{O} 1-\mathrm{V}-\mathrm{O} 5^{\mathrm{iii}}$ & $97.6(9)$ \\
\hline $\mathrm{O} 1-\mathrm{V}-\mathrm{O} 1^{\mathrm{iv}}$ & $177.2(5)$ & $\mathrm{V}-\mathrm{O} 1-\mathrm{V}^{\mathrm{i}}$ & $140.3(2)$ \\
\hline
\end{tabular}

Symmetry codes: (i) $1 / 2+x, 1 / 2-y, 1 / 2+z$; (ii) $1 / 2+x,-1 / 2+y, z$; (iii) $x, 1-y, 1 / 2+z$; (iv) $-1 / 2+x, 1 / 2-y,-1 / 2+z$.

After convergence, the pseudo-mirror constraints on the atomic coordinates were removed, followed by the $a=c$ constraint in the next step. Figure 1 shows a plot of the final Rietveld fit. The resulting crystal structure data are presented in the Tables I-III. The amount of $\beta-\mathrm{VOPO}_{4}$ as quantified from the fit was found to be $\sim 10 \%$.

Further details about the crystal structure investigation may be obtained from the Fachinformationszentrum Karlsruhe, D-76344 Eggenstein-Leopoldshafen, Germany (Fax: (+49)7247-808-666; E-mail: crysdata@fizkarlsruhe.de), by quoting the deposition number CSD415924 and article citation.

\subsection{Comparative model evaluation}

Due the fact that a different structure model had been published in the meantime [8], we had to test our Cc model in comparison to the $P 2_{1} / n$ model of Song et al. Overall, both models differ only slightly from each other and are equally chemically plausible. To ensure a fair comparison, we performed a series of comparative fits with corresponding pairs of models, using several levels of constraints, ranging from pseudo-mirror constraints as explained above, via free atomic coordinates but $a=c$, to a completely unconstrained refinement. Additionally, we included a scenario where the 200/002 reflection $\left(\sim 27.1^{\circ} 2 \theta\right)$ was excluded from the fit, as it appears that this was also the case in the original refinement of the $P 2_{1} / n$ model ( $c f$. Figure 1 of reference [8]). No preferred orientation model was used in any of the comparative fits, because such additional degree of freedom might be able to mask shortcomings of one or the other model. The results of the pairs of fits were compared with respect to the $R_{w p}$ values and the plausibility of the resulting geometry. 
In all scenarios, the Cc model yielded lower $R_{w p}$ values than the corresponding $P 2_{1} / n$ model. Moreover, even the lowest $R_{w p}$ obtained in the space group $P 2_{1} / n$ was still higher than any $R_{w p}$ in $C c$. The resulting geometry of the $C c$ model was always found to be less distorted than the corresponding $P 2_{1} / n$ version (Fig. 2 ). In addition, the geometry of the structure in the space group Cc proved to be much less sensitive towards changes of the refinement boundary conditions. At all levels of constraints, the Cc model was able to account for the intensity of the 200/002 reflection. In contrast, the $P 2_{1} / n$ model with pseudo-mirror constraints failed in this respect, which was also the case when the atoms were fixed to the coordinates published in reference [8]. The $P 2_{1} / n$ model was able to simulate the $200 / 002$ reflection only when all atomic coordinates were refined freely, which resulted in a rather distorted geometry (Fig. 2, upper right). Apparently, the direction of this distortion is the same (though slightly stronger) as in the "as published" geometry (Fig. 2, lower right). Thus, we can conclude that the response of the $P 2_{1} / n$ model towards refinement in our investigation is indeed representative.
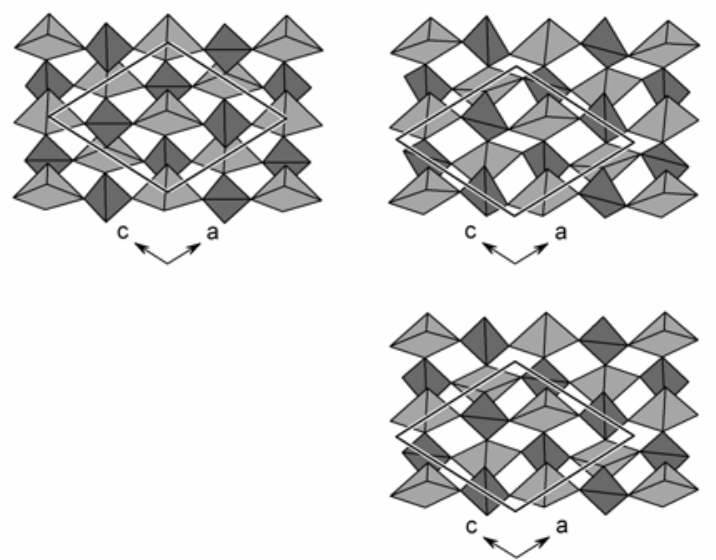

Figure 2: Comparison of the competing structure models for $\varepsilon-\mathrm{VOPO}_{4}$. The $C c$ model refined without constraints (top left) is much less distorted than the corresponding version of the $P 2_{1} / n$ model (top right). The distortion in the latter follows the same trends (though more exaggerated) that are visible in the $P 2_{1} / n$ structure as published in reference [8] (lower right). While all pyramids point into the same direction in the space group $C c$, they alternate in $P 2_{1} / n$.

\section{Discussion}

\subsection{Comparison of the $\varepsilon$-VOPO${ }_{4}$ structure models}

In vanadium $(\mathrm{V})$ oxidic structures, the vanadium atom usually features a strongly distorted "octahedral" coordination, with a very long $\mathrm{V}-\mathrm{O}$ distance in trans position to a rather short one. The latter is usually interpreted as a $\mathrm{V}=\mathrm{O}$ double bond (vanadyl bond), while the former may be regarded as an "intermolecular" short contact $\mathrm{V} \cdots \mathrm{O}$ rather than a true bond. Consequently, the coordination geometry of the vanadium atom may also be depicted as square pyramidal, though this simplification may obscure the fact that a sixth coordinating atom appears to be systematically present over the pyramid base. We will use both the "octahedral" and the "pyramidal representation" to discuss the structures, as we think that both views have certain benefits that complement each other. While the octahedral view shows infinite chains of trans-corner sharing $\mathrm{VO}_{6}$ octahedra in all $\mathrm{VOPO}_{4}$ polymorphs characterized so far, a corresponding pyramidal representation results in stacks of $\mathrm{VO}_{5}$ pyramids, with each pyramid apex pointing at the base of the next pyramid.

Crystallographically, as well as chemically, $\varepsilon-\mathrm{VOPO}_{4}$ can be derived from monoclinic $\mathrm{VPO}_{4} \cdot \mathrm{H}_{2} \mathrm{O}$. The latter phase crystallizes in the space group $\mathrm{C} 2 / \mathrm{c}$ and contains completely symmetric V-O-V chains, with the vanadium atom located at a center of inversion and the bridging oxygen atom on a twofold rotational axis (Wyckoff positions $4 c$ and $4 e$, respectively). Consequently, any symmetry reduction to the typical asymmetric $\mathrm{V}=\mathrm{O} \cdots \mathrm{V}$ chain in $\mathrm{VOPO}_{4}$ must involve the loss of these particular symmetry elements. The space groups $C c$ and $P 2_{1} / n$ both are subgroups of $C 2 / c$ which fulfill this requirement. The principal difference between the $C c$ and $P 2_{1} / n$ space group models for $\varepsilon-\mathrm{VOPO}_{4}$ is visible in the pyramidal representation (Fig. 2). In the $P 2_{1} / n$ model, neighboring stacks of $\mathrm{VO}_{5}$ pyramids alternate with respect to the direction of the pyramid apices (anti-parallel $\mathrm{V}=\mathrm{O} \cdots \mathrm{V}$ chains), a situation also found in $\beta-\mathrm{VOPO}_{4}$ (Fig. 3, top left). In contrast, the $C c$ structure model exhibits only stacks of pyramids pointing into the same direction (parallel $\mathrm{V}=\mathrm{O} \cdots \mathrm{V}$ chains).

It must be noted here that the variation in the orientation of the $\mathrm{V}=\mathrm{O} \cdots \mathrm{V}$ chains is indeed a small difference. For example, the orientation of the $\mathrm{VO}_{5}$ pyramids is the main distinction between $\alpha_{I^{-}}$and $\alpha_{I^{-}}-V^{-} P_{4}$ [17]. While the direction of the vanadyl bonds is obviously stable enough to separate $\alpha_{\mathrm{I}^{-}}$and $\alpha_{\mathrm{II}^{-}}-\mathrm{VOPO}_{4}$ as crystallographically defined phases, this situation can change upon chemical reaction. For instance, both chemical and electrochemical intercalation of lithium into $\alpha_{\mathrm{II}}-\mathrm{VOPO}_{4}$ will yield tetragonal $\mathrm{LiVOPO}_{4}$ with an $\alpha_{\mathrm{I}}$-type host lattice structure [18], i.e. the orientation of the $\mathrm{V}=\mathrm{O} \cdots \mathrm{V}$ chains is inverted during the reaction. This can be understood by realizing that the change from a $\mathrm{V}=\mathrm{O} \cdots \mathrm{V}$ to a $\mathrm{V} \cdots \mathrm{O}=\mathrm{V}$ configuration can be accomplished without any bond breaking by small displacements of the atoms involved. Consequently, it might be possible that both $C c$ and $P 2_{1} / n$ versions of $\varepsilon-\mathrm{VOPO}_{4}$ could exist as crystallographically distinct phases. Alternatively, the real structure of $\varepsilon-\mathrm{VOPO}_{4}$ could be composed of a statistical mixture resulting from "intergrowth" of these two ideal structure variants. However, the results of this study, especially concerning the reproduction of the $200 / 002$ reflection and the distortion tendencies observed in the $P 2{ }_{1} / n$ model, consistently indicate that the Cc structure model is a better approximation to the real structure of $\varepsilon-\mathrm{VOPO}_{4}$ than the $P 2_{1} / n$ version. 


\subsection{Comparison of $\varepsilon-\mathrm{VOPO}_{4}$ to related structures}

The octahedral view emphasizes the relationships between $\varepsilon-\mathrm{VOPO}_{4}$ and $\beta-\mathrm{VOPO}_{4}$ on the one hand, and $\varepsilon-\mathrm{VOPO}_{4}$ and monoclinic $\mathrm{VPO}_{4} \cdot \mathrm{H}_{2} \mathrm{O}$ on the other (Fig. 3). Both $\mathrm{VOPO}_{4}$ polymorphs share essentially the same "building blocks", but "stacked" in a different way, resulting in a different connectivity pattern. The connectivity pattern in turn is shared between $\varepsilon-\mathrm{VOPO}_{4}$ and monoclinic $\mathrm{VPO}_{4} \cdot \mathrm{H}_{2} \mathrm{O}$. In other words, the structures of $\varepsilon-\mathrm{VOPO}_{4}$ and monoclinic $\mathrm{VPO}_{4} \cdot \mathrm{H}_{2} \mathrm{O}$ are indeed united by a topotactic relationship, as was first proposed by Lim et al. based on a hydrogen spillover experiment [4]. In analogy, it seems probable that the "new phase" reported in the same article (cf. Figure 9 of reference [4]), which was obtained from hydrogen spillover reduction of $\beta-\mathrm{VOPO}_{4}$, is a new polymorph of $\mathrm{VPO}_{4} \cdot \mathrm{H}_{2} \mathrm{O}$ with the same connectivity as in $\beta-\mathrm{VOPO}_{4}$.
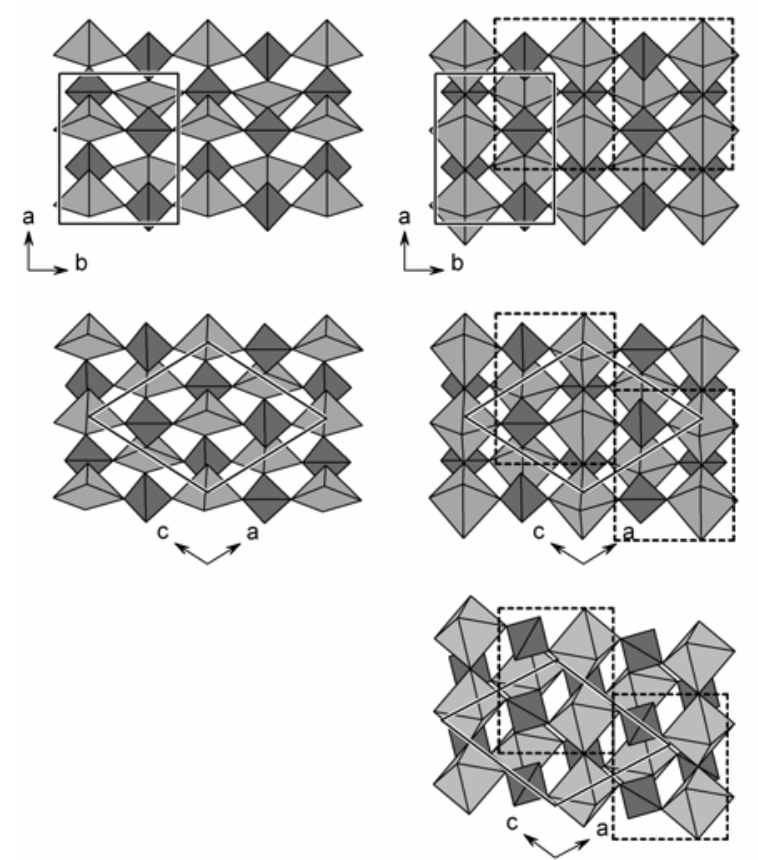

Figure 3: Comparison of the crystal structures of $\beta-\mathrm{VOPO}_{4}$ (top row), $\varepsilon-\mathrm{VOPO}_{4}$ (center row) and monoclinic $\mathrm{VPO}_{4} \cdot \mathrm{H}_{2} \mathrm{O}$ (bottom). The pyramidal representation (left column) reveals the difference in orientation of the $\mathrm{V}=\mathrm{O}$ bonds between $\beta$ - and $\varepsilon$-VOPO 4 . The octahedral representation (right column) emphasizes the common building blocks (dashed boxes) in $\beta$ - and $\varepsilon$-VOPO${ }_{4}$, as well as the connectivity pattern shared between $\varepsilon-\mathrm{VOPO}_{4}$ and monoclinic $\mathrm{VPO}_{4} \cdot \mathrm{H}_{2} \mathrm{O}$.

Among the V(IV) compounds $\mathrm{MVOPO}_{4}(\mathrm{M}=$ alkali metal or $\mathrm{NH}_{4}$ ), only representatives with small alkali metal ions $(\mathrm{M}=\mathrm{Li}, \mathrm{Na})$ follow the same structural trends seen in the $\mathrm{VOPO}_{4}$ polymorphs, featuring trans-corner sharing $\mathrm{VO}_{6}$ octahedra [19]. Triclinic $\alpha-\mathrm{LiVOPO}_{4}$ [20] and the very similar monoclinic $\mathrm{NaVOPO}_{4}[21,22]$ show the same connectivity as $\varepsilon-\mathrm{VOPO}_{4}$, while orthorhombic $\beta$-LiVOPO
[23] corresponds to $\beta-\mathrm{VOPO}_{4}$. The recently described tetragonal polymorph of $\mathrm{LiVOPO}_{4}$ contains an $\alpha_{\mathrm{I}}-\mathrm{VOPO}_{4}$ type host lattice [18] (note that Dupré et al. therefore termed this phase " $\alpha_{\mathrm{I}}-\mathrm{LiVOPO}_{4}$ ", which should not be confused with the established term $\alpha-\mathrm{LiVOPO}_{4}$ for triclinic $\mathrm{LiVOPO}_{4}$ ).

Unlike the parallel oriented $\mathrm{VO}_{5}$ pyramid stacks contained in the $\varepsilon-\mathrm{VOPO}_{4}$ structure proposed here, $\alpha-\mathrm{LiVOPO}_{4}\left(\right.$ like $\mathrm{NaVOPO}_{4}$ ) exhibits anti-parallel stacks. Consequently, every second $\mathrm{V}=\mathrm{O} \cdots \mathrm{V}$ chain has to be inverted upon intercalation of one equivalent lithium into $\varepsilon-\mathrm{VOPO}_{4}$. As pointed out above, inversion of the vanadyl bonds due to lithium intercalation has been observed before. Thus, the $\mathrm{VOPO}_{4}$ and corresponding $\mathrm{LiVOPO}_{4}$ polymorphs characterized so far represent examples spanning the whole range from no inversion $\left(\beta-\mathrm{VOPO}_{4} \rightarrow\right.$ $\beta$-LiVOPO ${ }_{4}$ and $\alpha_{\mathrm{I}}-\mathrm{VOPO}_{4} \rightarrow$ tetragonal $\mathrm{LiVOPO}_{4}$ ) via partial inversion $\left(\varepsilon-\mathrm{VOPO}_{4} \rightarrow \alpha-\mathrm{LiVOPO}_{4}\right)$ to complete inversion $\left(\alpha_{\mathrm{II}}-\mathrm{VOPO}_{4} \rightarrow\right.$ tetragonal $\left.\mathrm{LiVOPO}_{4}\right)$. It seems plausible that the easy inversion of vanadyl bonds offers a mechanism which allows the $\mathrm{VOPO}_{4}$ host lattice to fineadjust for the accommodation of the lithium ions. This additional flexibility in the lattice could be an important factor contributing to the interesting electrochemical properties of the $\mathrm{VOPO}_{4}$ family.

\section{Summary and conclusion}

We have presented a new structure model for $\varepsilon-\mathrm{VOPO}_{4}$ in the space group Cc. This structure exhibits stacks of $\mathrm{VO}_{5}$ pyramids with parallel orientation, as opposed to a previously published model (space group $P 2_{1} / n$ ) and $\beta-\mathrm{VOPO}_{4}$, which both show an anti-parallel pattern. It was found that the $C c$ model performs significantly better in terms of agreement between simulated and measured diffraction pattern, as well as in stability and plausibility of the resulting geometry.

The fact that this structure was successfully solved without prior knowledge of the correct space group demonstrates the potential oft the rigid body approach for structure determination from powder diffraction data in direct space. This strategy reduces the number of free parameters significantly, thus speeding up and stabilizing the refinement process and enhancing the discrimination between geometrically reasonable and unreasonable solutions. Provided that the chemical context allows for a fairly good prediction of local geometries, the rigid body approach offers a way to screen through a number of candidate space groups within much shorter time. 


\section{References}

[1] E. Bordes, P. Courtine, J. Chem. Soc., Chem. Commun. (1985) 294.

[2] G.J. Hutchings, A. Desmartin-Chomel, R. Olier, J.-C. Volta, Nature 368 (1994) 41.

[3] see e.g. G.W. Coulston, S.R. Bare, H. Kung, K. Birkeland, G.K. Bethke, R. Harlow, N. Herron, P.L. Lee, Science 275 (1997) 191, and references therein.

[4] S.C. Lim, J.T. Vaughey, W.T.A. Harrison, L.L. Dussac, A.J. Jacobson, J.W. Johnson, Solid State Ionics 84 (1996) 219.

[5] T.A. Kerr, J. Gaubicher, L.F. Nazar, Electrochem. Solid-State Lett. 3 (2000) 460.

[6] B.M. Azmi, T. Ishihara, H. Nishiguchi, Y. Takita, Electrochim. Acta 48 (2002) 165.

[7] B.M. Azmi, T. Ishihara, H. Nishiguchi, Y. Takita, J. Power Sources 119-121 (2003) 273.

[8] Y. Song, P.Y. Zavalij, M.S. Whittingham, J. Electrochem. Soc. 152 (2005) A721.

[9] R. Gopal, C. Calvo, J. Solid State Chem. 5 (1972) 432.

[10] J.T. Vaughey, W.T.A. Harrison, A.J. Jacobson, Inorg. Chem. 33 (1994) 2481.

[11] F.J.C. Sanchez, R.P.K. Wells, C. Rhodes, J.K. Bartley, C.J. Kiely, G.J. Hutchings, Phys. Chem. Chem. Phys. 3 (2001) 4122 .
[12] Topas v2.1, copyright 1999, 2000 Bruker AXS

[13] A.A. Coelho, J. Appl. Cryst. 33 (2000) 899.

[14] Platon for Windows Taskbar v1.081, copyright 1995-2005 L.J. Farrugia, University of Glasgow, Glasgow, UK

[15] PLATON, A Multipurpose Crystallographic Tool, v200905, copyright 1980-2005 A.L. Spek, Utrecht University, Utrecht, The Netherlands

[16] Diamond v3.1, copyright 1997-2005 Crystal Impact GbR, Bonn, Germany

[17] M. Tachez, F. Theobald, E. Bordes, J. Solid State Chem. 40 (1981) 280

[18] N. Dupré, G. Wallez, J. Gaubicher, M. Quarton, J. Solid State Chem. 177 (2004) 2896.

[19] F. Berrah, A. Guesdon, A. Leclaire, M.-M. Borel, J. Provost, B. Raveau, Solid State Sci. 3 (2001) 477.

[20] A.V. Lavrov, V.P. Nikolaev, G.G. Sadikov, M.A. PoraiKoshits, Phys. Dokl. 27 (1982) 680.

[21] L. Benhamada, A. Grandin, M.M. Borel, A. Leclaire, B. Raveau, C.R. Acad. Sci. Ser. II 314 (1992) 585.

[22] K.H. Lii, C.H. Li, T.M. Chen, S.L. Wang, Z. Krist. 197 (1991) 67.

[23] K.H. Lii, C.H. Li, C.Y. Cheng, S.L. Wang, J. Solid State Chem. 95 (1991) 35. 